\title{
Bankruptcy Problem Allocations and the Core of Convex Games
}

\author{
William Olvera-Lopez, ${ }^{1}$ Francisco Sanchez-Sanchez, ${ }^{2}$ and Iván Tellez-Tellez ${ }^{2}$ \\ ${ }^{1}$ School of Economics, UASLP, Avenida Pintores S/N, 78213 San Luis Potosí, SLP, Mexico \\ ${ }^{2}$ CIMAT, A.C., Jalisco S/N, 36240 Guanajuato, GTO, Mexico
}

Correspondence should be addressed to William Olvera-Lopez; william@cimat.mx

Received 18 March 2014; Revised 30 May 2014; Accepted 5 June 2014; Published 25 June 2014

Academic Editor: Jordi Massó

Copyright (C) 2014 William Olvera-Lopez et al. This is an open access article distributed under the Creative Commons Attribution License, which permits unrestricted use, distribution, and reproduction in any medium, provided the original work is properly cited.

A well-known result related to bankruptcy problems establishes that a vector is a bankruptcy allocation if and only if it belongs to the core of the associated O'Neill's bankruptcy game. In this paper we show that this game is precisely the unique TU-game based on convex functions that satisfies the previous result. In addition, given a bankruptcy problem, we show a way for constructing bankruptcy games such that the set of bankruptcy allocations is a subset of their core or their core is a subset of the set of bankruptcy allocations. Also, we show how these results can be applied for finding new bankruptcy solutions.

\section{Introduction}

There are several situations when an estate is disputed by a set of agents, each one of them claiming a part of the estate. The problem arises when the total claim is greater than the estate, giving place to a bankruptcy problem. Typically, in a bankruptcy allocation, an agent must receive at least zero and at most his claim. This is the kind of problems we study in this paper. The bankruptcy problem was introduced by O'Neill [1] and later studied by several authors. See [2-5] for some seminal papers and analysis on this topic.

Under the normative approach to bankruptcy problems, the aim is to find fair bankruptcy solutions, and two methods for this purpose are identified. The direct method, introduced by Young [6], comes from an axiomatic study of bankruptcy solutions based on the original problems. In the indirect method, one considers transferable utility cooperative games (TU-games) that summarize appropriately the conflict of the bankruptcy problem. Such games are now called bankruptcy games and were first proposed by O’Neill [1]. The main idea of finding a solution in both settings is to give a rule (a bankruptcy solution) for distributing the estate among the agents satisfying uniquely a set of desirable properties: the interpretation of a bankruptcy solution is the recommendation of an external agent or arbitrator. A vast literature follows both approaches, partially gathered by Thomson [4]. The present work is based on the indirect approach. Particularly, given a bankruptcy problem, we construct a TU-game for every increasing-continuous function defined in a certain real interval.

For a fixed bankruptcy problem, we study TU-games where the characteristic function value for each coalition depends on a function of the agents' claims in that coalition, following the idea of the bankruptcy game proposed by O'Neill [1]. In [2], it is shown that, given a bankruptcy problem, the core of O'Neill's bankruptcy game is exactly the set of bankruptcy allocations. So, it seems tempting to ask if there exists another bankruptcy game, based on a convex function, with this property. Another TU-game with this property could help us to have a better understanding of the possible allocations for a bankruptcy problem. We prove that O'Neill's bankruptcy game is the only one with this property. To give a complement to our treatment of the problem, we provide two sets of TU-games: in the first one, each game has a core of allocations to the bankruptcy problem but not the converse; for every game in the second set, every allocation of the bankruptcy problem is in its core. Finally, we show how these last results can be applied to find new bankruptcy solutions.

If we use TU-game theoretic results to find allocations of a bankruptcy problem, it is desirable that the proposed allocation belongs to the core of the corresponding TUgame. If an allocation is not in the core of the associated TU-game, it means that there exists a subgroup of agents such that the amount assigned to them is lower than their 
amount according to the TU-game (the allocation does not satisfy group rationality). Thus, is it more reasonable that the bankruptcy allocation proposed by a TU-game solution belongs to the core of the game? That is the main reason we are interested in using TU-games with a nonempty core for solving bankruptcy problems. Contrary to our expectation, there is just one TU-game based on convex functions with the additional property that its core and the set of bankruptcy allocations coincide. We show a way for finding TU-games such that all the elements of their cores are allocations for the original bankruptcy problem, and we show how this result can be applied for proposing new bankruptcy solutions.

The paper is organized as follows. In Section 2 we introduce the notation and some basic definitions related to the standard bankruptcy problem as well as transferable utility cooperative game theory concepts. In Section 3 we present our main results and illustrate them with some examples. Finally, in Section 4 we show an application of our results for finding bankruptcy solutions.

\section{Notation and Definitions}

Given a set of agents $N=\{1, \ldots, n\}$, a bankruptcy problem $(E ; c)$ consists of a number $E \in \mathbb{R}_{+}$, the estate, and a claims vector $c \in \mathbb{R}^{n}$ where $c_{i}>0$ for all $i \in N$ and the total claim, $C$, satisfies $C:=c_{1}+\cdots+c_{n}>E$. We denote the set of all possible bankruptcy problems with a set of agents $N$ by $B_{N}$. This kind of problem is interpreted as a dispute between claimers, where the $i$ th claimer demands a quantity $c_{i}$, from a fixed amount, $E$, which is not enough to satisfy all demands.

Given a bankruptcy problem $B=(E ; c)$ a bankruptcy allocation for $B$ is a vector $x \in \mathbb{R}^{n}$ such that

$$
\sum_{i \in N} x_{i}=E x \quad 0 \leq x_{i} \leq c_{i}, \forall i \in N
$$

A solution for bankruptcy problems is a function $\varphi: B_{N} \rightarrow$ $\mathbb{R}^{n}$ such that $\varphi(B)$ is a bankruptcy allocation. In a bankruptcy allocation, the estate is totally allocated and each agent obtains a positive amount not exceeding his claim.

For $x \in \mathbb{R}^{n}$ and $S \subseteq N$, let $x(S):=\sum_{i \in S} x_{i}$. Notice that for a bankruptcy problem $(E ; c), C=x(N)$. Next, we present some standard notions of cooperative game theory which will be used in what follows. A transferable utility cooperative game, a TU-game, is defined as a pair $(N, v)$, where $N=\{1, \ldots, n\}$ is the set of players and $v: 2^{N} \rightarrow \mathbb{R}$, and is the characteristic function evaluated at the $2^{N}$ possible subsets of $N$ with $v(\emptyset)=$ 0 . Given a coalition $S \subseteq N, v(S)$ is interpreted as the worth the agents in coalition $S$ can obtain for their cooperation.

Let $G_{N}$ be the set of TU-games with set of players $N$. Given a TU-game $(N, v)$ its dual game, $\left(N, v^{*}\right)$, is defined by $v^{*}(S)=v(N)-v(N \backslash S)$ for every $S \subseteq N$. A solution for a TUgame is a multivalued map $\phi: G_{N} \rightarrow \mathbb{R}^{n}$, where $\phi_{i}(N, v)$ is the amount that each agent obtains in the game $(N, v) \in G_{N}$. There are several solutions for TU-games; see, for example,
$[7,8]$. In particular, one can consider the core of $(N, v)$ which is given by the set

$$
\begin{aligned}
\mathscr{C} & (N, v) \\
& =\left\{x \in \mathbb{R}^{n} \mid x(S) \geq v(S) \forall S \subsetneq N, x(N)=v(N)\right\} .
\end{aligned}
$$

Considering nonemptiness, the core contains all allocations in which each coalition $S \in 2^{N}$ obtains at least its own worth and the amount $v(N)$ is distributed amongst all agents. An allocation $x$ satisfying the last condition, $x(N)=v(N)$, is called efficient.

A TU-game $(N, v) \in G_{N}$ is called convex if for every player $i \in N$ and each pair of coalitions $S, T$ such that $S \subseteq$ $T \subseteq N \backslash\{i\}$ the next condition holds:

$$
v(T \cup\{i\})-v(T) \geq v(S \cup\{i\})-v(S) .
$$

It is known that if $(N, v)$ is convex then it has a nonempty core [9].

There is a vast literature where bankruptcy problems and TU-games are related in some way. O'Neill [1] proposed a TUgame for studying bankruptcy problems; with his model he summarized the conflict in an appropriated form. In general, a TU-game with this characteristic is called a bankruptcy game. O'Neill's bankruptcy game is as follows, for every problem $B=(E ; c)$ :

$$
v^{B}(S)=\max \left\{0, E-\sum_{i \in N \backslash S} c_{i}\right\}, \quad \forall s \subseteq N .
$$

The idea of this game is to assign as the worth of a coalition $S \subseteq N$ the remainder estate after satisfying the claims of the agents on $N \backslash S$. Curiel et al. [2] showed that the bankruptcy game proposed by O'Neill is convex and, additionally, that the core of O'Neill's bankruptcy game is exactly the set of all bankruptcy allocations.

\section{Results}

Let $B=(E ; c) \in B_{N}$ be a bankruptcy problem and consider the next set of functions:

$\mathscr{F}^{B}=\{f \mid f:[0, C] \longrightarrow[0, E]$ increasing-continuous with

$$
f(0)=0, f(C)=E\} \text {. }
$$

In all what follows we consider that the problem $B=(E ; c)$ and the set $\mathscr{F}^{B}$ are given and fixed.

For $f \in \mathscr{F}^{B}$, the dual function is defined by

$$
f^{*}(\tau):=f(C)-f(C-\tau), \quad \tau \in[0, C] .
$$

An easy reference to $f^{*}$ is provided by its graph: it is the graph of $f$ rotated with respect to the line $\ell(\tau)=E \tau / C$ (the positive diagonal of the square $[0, C] \times[0, E]$ in $\mathbb{R}^{2}$ ).

For every $f \in \mathscr{F}^{B}$, we define the TU-game $\left(N, v_{f}\right)$ associated with $f$ as

$$
v_{f}(S):=f\left(\sum_{i \in S} c_{i}\right), \quad \forall S \subseteq N .
$$


We are interested in the core of certain TU-games $\left(N, v_{f}\right)$. For this purpose, we focus on a kind of TU-games with a nonempty core.

Lemma 1. For every bankruptcy problem $B=(E ; c)$, if $f \in \mathscr{F}^{B}$ is a convex function, then $\left(N, v_{f}\right)$ is a convex TU-game.

We establish another useful result related to the set $\mathscr{F}^{B}$.

Lemma 2. For every bankruptcy problem $B=(E ; c)$ and every function $f \in \mathscr{F}^{B}$,

(1) $f^{*} \in \mathscr{F}^{B}$ and $\left(f^{*}\right)^{*}=f$;

(2) $v_{f^{*}}=v_{f}^{*}$;

(3) if $f$ is a convex function, then $f^{*}$ is concave; if $f$ is concave, then $f^{*}$ is convex;

(4) if $g \in \mathscr{F}^{B}$ then $f(\tau) \leq g(\tau)$ if and only if $f^{*}(\tau) \geq$ $g^{*}(\tau)$, and $f(\tau) \geq g(\tau)$ if and only if $f^{*}(\tau) \leq g^{*}(\tau)$, for every $\tau \in[0, C]$.

For the proofs of the previous lemmas, please refer to Appendix 4.

The next proposition is referred to Curiel et al. [2].

Proposition 3. $x \in \mathbb{R}^{n}$ is a bankruptcy allocation for $(E ; c)$ if and only if $x \in \mathscr{C}(N, w)$, where the TU-game $(N, w)$ is defined as follows:

$$
w(S)=\max \left\{0, E-\sum_{i \in N \backslash S} c_{i}\right\}, \quad \forall S \subseteq N .
$$

Example 4. Let us consider a bankruptcy problem with $n=3$, $c=(4,6,20)$, and $E=26$. For this situation, the TU-game $(N, w)$ is as follows:

$$
\begin{aligned}
& w(\{1\})=0 ; \quad w(\{2\})=2 ; \quad w(\{3\})=16 ; \\
& w(\{1,2\})=6 ; \quad w(\{1,3\})=20 ; \quad w(\{2,3\})=22 ; \\
& w(N)=26 .
\end{aligned}
$$

The core of this game is the set

$$
\begin{gathered}
\mathscr{C}(N, w)=\left\{x \in \mathbb{R}^{n} \mid x_{1} \in[0,4], x_{2} \in[2,6], x_{3} \in[16,20],\right. \\
\left.x_{1}+x_{2}+x_{3}=26\right\} .
\end{gathered}
$$

As Proposition 3 establishes, this set contains the whole set of bankruptcy allocations for its corresponding bankruptcy problem. It is depicted in Figure 1 into the set of efficient solutions of $(N, w)$.

Now, given a bankruptcy problem $B=(E ; c)$, we consider the next set of functions:

$$
\mathscr{F}_{*}^{B}=\left\{f \in \mathscr{F}^{B}: f \text { is convex }\right\},
$$

and the related games

$$
\mathscr{V}^{B}=\left\{v_{f}: f \in \mathscr{F}_{*}^{B}\right\} .
$$

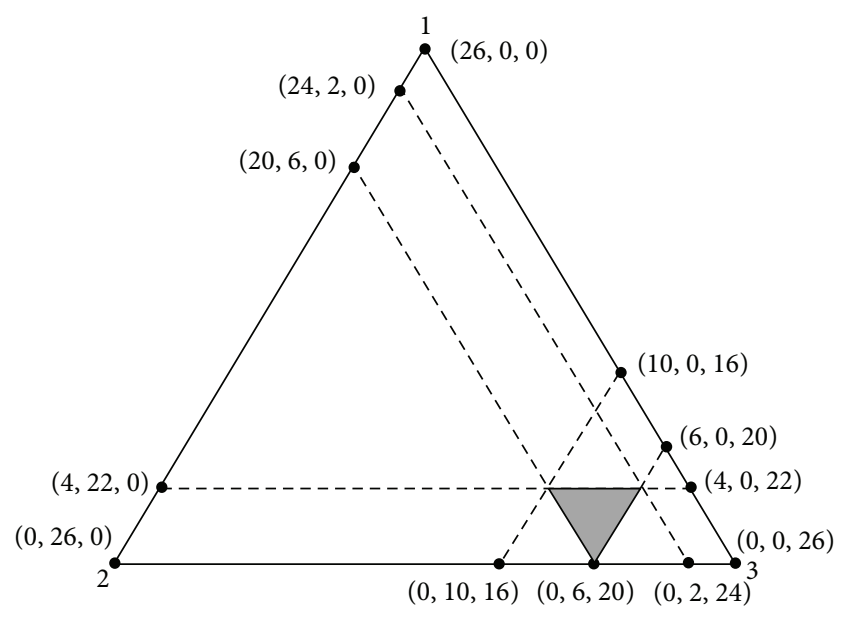

FIGURE 1: Barycentric representation of efficient solutions for the TU-game $(N, w)$ associated with the bankruptcy problem $[26 ;(4,6$, 20)]. The upper vertex corresponds to Agent 1, the lower-left to Agent 2, and the lower-right to Agent 3. The grey area is $\mathscr{C}(N, w)$.

We will show that a TU-game $(N, v)$, with $v \in \mathscr{V}^{B}$, satisfies Proposition 3 if and only if it is O'Neill's game $(N, w)$. The notation $(N, w)$, or simply $w$, is maintained in the whole text to make reference to this special game.

Theorem 5. Given a bankruptcy problem $B=(E ; c)$, the core of a TU-game $\left(N, v_{f}\right)$, with $v_{f} \in \mathscr{V}^{B}$, is exactly the set of allocations for the bankruptcy problem if and only if $v_{f}=w$.

Proof. We will prove it by contradiction. If $v_{f} \in \mathscr{V}^{B}$ for certain $f \in \mathscr{F}^{B}$, with $v_{f} \neq w$ also satisfying Proposition 3, then there exists a coalition $S \subset N$ such that $v_{f}(S) \neq w(S)$.

Let us define the function $g \in \mathscr{F}^{B}$ :

$$
g(\tau)=\max \{0, E-C+\tau\} .
$$

Notice that $w(S)=g(c(S))$ for all $S \subseteq N$. On one hand, if $v_{f}(S)<w(S)$, then $f(c(S))<g(c(S))$ and, due to the monotonicity of $f$, we have

$$
C-E<c(S)<C .
$$

This inequality implies that there exists at least one $k \epsilon$ $N \backslash S$ such that $c_{k}<E$. For this agent $k$, the next condition holds:

$$
C-E<c(S) \leq C-c_{k}<C,
$$

and, because of the monotonicity and convexity of $f$ and $g$, we have $f\left(C-c_{k}\right)<g\left(C-c_{k}\right)$ because $f(c(S))<g(c(S))$.

According to Lemma $1, v_{f}$ is a convex game and then, $\mathscr{C}\left(N, v_{f}\right) \neq \varnothing$. A well-known result establishes that if a TUgame $(N, v)$ is convex, then for every permutation $\sigma$ of $N$ the allocation $x^{\sigma} \in \mathbb{R}^{n}$, whose coordinates are

$$
x_{i}^{\sigma}(v)=v(\sigma(1), \ldots, \sigma(i))-v(\sigma(1), \ldots, \sigma(i-1)),
$$


is in the core of the game. In particular, for the agent $k$ defined previously and the TU-game $\left(N, v_{f}\right)$, the $k$ th coordinate of some of these vectors satisfies

$$
\begin{aligned}
x_{k} & =v_{f}(N)-v_{f}(N \backslash k)=E-f\left(C-c_{k}\right) \\
& >E-g\left(C-c_{k}\right)=c_{k} .
\end{aligned}
$$

It follows that $x$ is not a solution to the bankruptcy problem $(E ; c)$ and, then, we have reached a contradiction.

On the other hand, if $v_{f}(S)>w(S)$ there exists a subset $S \subset N$ such that

$$
f(c(S))>g(c(S)) .
$$

The continuity of $f$ and $g$ ensures that there exists an interval $(\alpha, \beta)$ where $f(\tau)>g(\tau)$ for every $\tau \in(\alpha, \beta)$. This fact, in addition with the convexity of $f$ implies that $C-E \in(\alpha, \beta)$.

If

$$
\alpha<c(S) \leq C-E<\beta,
$$

then $E \leq c(N \backslash S)$. So, an allocation with $x_{i}=0$ for each $i \in S$ and $x(N \backslash S)=E$ is not in the core of $\left(N, v_{f}\right)$ because

$$
v_{f}(S)=f(c(S))>g(c(S))=x(S)=0 .
$$

Finally, if

$$
\alpha<C-E<c(S)<\beta,
$$

then $E>c(N \backslash S)$. So, an allocation such that $x_{i}=c_{i}$ for every $i \in N \backslash S$ and $x(S)=E-c(N \backslash S)$ is not in the core of $\left(N, v_{f}\right)$ because

$$
v_{f}(S)=f(c(S))>g(c(S))=E-c(N \backslash S)=x(S),
$$

obtaining, again, a contradiction.

In our last result we find two sets of convex TU-games; in the first one, the elements of their cores are allocations to a bankruptcy problem $(E ; c)$; in the second set, those allocations are elements of their cores.

We recall the concept of dual-core of a TU-game $(N, v)$ :

$$
\begin{aligned}
& \widehat{\mathscr{C}}(N, v)=\left\{x \in \mathbb{R}^{n}: x(N)=v(N),\right. \\
&x(S) \leq v(S) \text { for every } S \subset N\} .
\end{aligned}
$$

A related result about the relation between the core and the dual-core establishes that $x \in \mathscr{C}(N, v)$ if and only if $x \in$ $\widehat{\mathscr{C}}\left(N, v^{*}\right)$ for every TU-game $(N, v)$.

Theorem 6. For every bankruptcy problem $B=(E ; c)$, let us consider

$$
\begin{aligned}
& \mathscr{V}_{1}^{B}=\left\{v_{f} \in \mathscr{V}^{B}: \frac{E \tau}{C} \geq f(\tau) \geq g(\tau), \tau \in[0, C]\right\}, \\
& \mathscr{V}_{2}^{B}=\left\{v_{f} \in \mathscr{V}^{B}: g(\tau) \geq f(\tau), \tau \in[0, C]\right\},
\end{aligned}
$$

with $g$ defined as in (13). If $v_{f} \in \mathscr{V}_{1}^{B}$, then each element in the core of $\left(N, v_{f}\right)$ is an allocation for the problem $(E ; c)$. If $v_{f} \in$ $\mathscr{V}_{2}^{B}$ then every bankruptcy allocation of $(E ; c)$ belongs to the core of $\left(N, v_{f}\right)$.
Proof. Every TU-game $\left(N, v_{f}\right)$, with $v_{f} \in \mathscr{V}_{j}^{B}(j=1,2)$, is convex since $f$ is a convex function. Then, it has a nonempty core. If $x \in \mathscr{C}\left(N, v_{f}\right)$ with $v_{f} \in \mathscr{V}_{1}^{B}$, then $x(N)=v_{f}(N)=E$ and

$$
0 \leq x_{i} \leq E-f(c(N \backslash\{i\})) \leq E-\max \left\{0, E-c_{i}\right\} \leq c_{i}
$$

for every $i \in N$. So, $x$ is a bankruptcy allocation.

If $v_{f} \in \mathscr{V}_{2}^{B}$ and $x$ is a bankruptcy allocation, then $x(N)=$ $E=v_{f}(N)$ and $x(S) \leq c(S)$ for every $S \subset N$. Thus,

$$
x(S) \leq \min \{E, c(S)\}=g^{*}(c(S)) \leq f^{*}(c(S)) .
$$

That is, $x \in \widehat{\mathscr{C}}\left(N, v_{f^{*}}\right)$ and, recalling Lemma $2, x \in \widehat{\mathscr{C}}\left(N, v_{f}^{*}\right)$. Then, $x \in \mathscr{C}\left(N, v_{f}\right)$.

Example 7. Let $(E ; c)$ be the bankruptcy problem of Example 4. In this case, the TU-game $(N, w)$ referred to in Proposition 3 is $w(S)=\max \{0, c(S)-1\}$ for every $S \subseteq N$, arising from the convex function $g_{0}(\tau)=\max \{0, \tau-1\}$. For $g_{1}$ defined as

$$
g_{1}(\tau)= \begin{cases}\frac{2}{5} \tau, & \text { if } \tau<5, \\ \frac{24}{25}(\tau-5)+2, & \text { if } \tau \geq 5,\end{cases}
$$

we have $v_{g_{1}} \in \mathscr{V}_{1}^{B}$ as follows:

$$
\begin{gathered}
v_{g_{1}}(\{1\})=1.6 ; \quad v_{g_{1}}(\{2\})=2.96 ; \quad v_{g_{1}}(\{3\})=16.4 ; \\
v_{g_{1}}(\{1,2\})=6.8 ; \quad v_{g_{1}}(\{1,3\})=20.24 ; \\
v_{g_{1}}(\{2,3\})=22.16 ; \quad v_{g_{1}}(N)=26 .
\end{gathered}
$$

The core of $\left(N, v_{g_{1}}\right)$ contains all the efficient vectors $\left(x_{1}, x_{2}\right.$, $x_{3}$ ) such that

$$
\begin{gathered}
x_{1} \in[1.6,3.84] ; \quad x_{2} \in[2.96,5.76], \\
x_{3} \in[16.4,19.2] .
\end{gathered}
$$

Clearly, $\mathscr{C}\left(N, v_{g_{1}}\right) \subset \mathscr{C}(N, w)$. So, every element in $\mathscr{C}\left(N, v_{g_{1}}\right)$ is a bankruptcy allocation for $(E ; c)$, but there exists more.

On the other hand, for the next function $g_{2}$

$$
g_{2}(\tau)= \begin{cases}0, & \text { if } 0 \leq \tau<4, \\ \frac{1}{2}(\tau-4), & \text { if } 4 \leq \tau<20, \\ \frac{9}{5}(\tau-20)+8, & \text { if } \tau \geq 20,\end{cases}
$$

we have $v_{g_{2}} \in \mathscr{V}_{2}^{B}$ as follows:

$$
\begin{gathered}
v_{g_{2}}(\{1\})=0 ; \quad v_{g_{2}}(\{2\})=1 ; \quad v_{g_{2}}(\{3\})=8 ; \\
v_{g_{2}}(\{1,2\})=3 ; \quad v_{g_{2}}(\{1,3\})=15.20 ; \\
v_{g_{2}}(\{2,3\})=18.80 ; \quad v_{g_{2}}(N)=26 .
\end{gathered}
$$




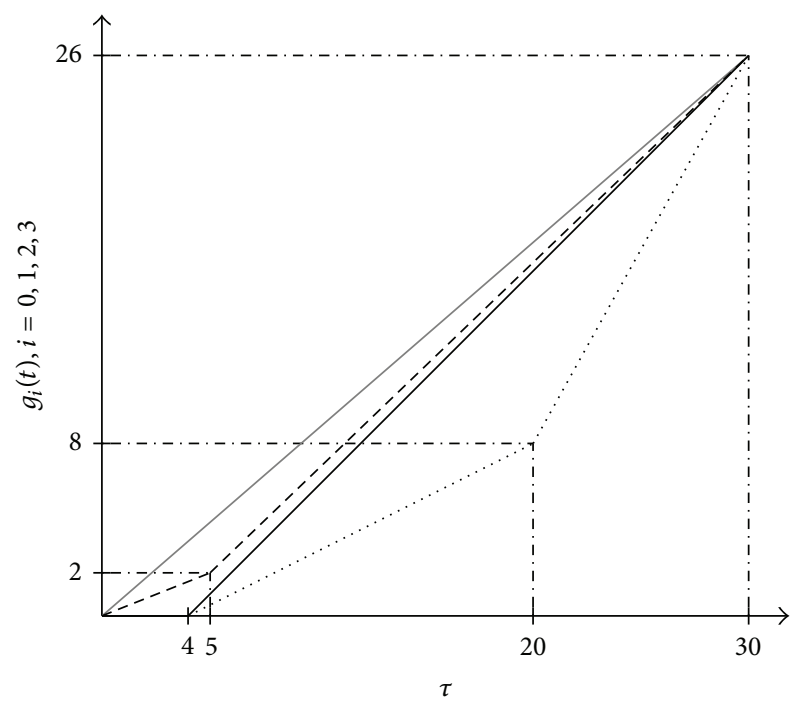

FIgURE 2: Graphics of the functions in Example 7. The solid black line corresponds to $g_{0}$, the dashed to $g_{1}$, and the dotted to $g_{2}$. The gray graphic corresponds to the function $g_{3}(\tau)=26 \tau / 30$.

In this case, $\mathscr{C}\left(N, v_{g_{2}}\right)$ contains all the efficient vectors $\left(x_{1}, x_{2}, x_{3}\right)$ such that

$$
x_{1} \in[0,7.2] ; \quad x_{2} \in[1,10.8], \quad x_{3} \in[8,23] .
$$

Now, we can easily check that $\mathscr{C}(N, w) \subset \mathscr{C}\left(N, v_{g_{2}}\right)$, verifying Theorem 6 for the bankruptcy problem in Example 4 and functions $g_{1}$ and $g_{2}$ defined previously. In Figure 2, the functions $g_{0}, g_{1}$, and $g_{2}$ are depicted.

\section{An Application}

In this section we apply the results of Theorem 6 to find bankruptcy allocations belonging to the core of certain TUgames.

Given a bankruptcy problem $B=(E ; c)$, let

$$
\mathscr{W}^{B}=\left\{v_{f_{\alpha}}: f_{\alpha}(\tau)=\max \{0, E-\alpha(C-\tau)\}, \alpha \in\left[\frac{E}{C}, 1\right]\right\} .
$$

For every $\alpha$, each TU-game $\left(N, v_{f_{\alpha}}\right)$ in $\mathscr{W}^{B}$ can be considered as a special case of the bankruptcy game introduced by O'Neill [1]. When $\alpha=1,\left(N, v_{f_{1}}\right)$ is precisely that TU-game is the function $f_{1}=g$, with $g$ defined as in (13). Moreover, from Theorem 6 , the minimum value of $\alpha$ that ensures $\left(N, v_{f_{\alpha}}\right)$ does not have an empty core is $\alpha=E / C$. So, $\mathscr{W}^{B} \subseteq \mathscr{V}_{1}^{B}$ contains all possible generalizations of O'Neill's bankruptcy games with a nonempty core.

Let $\varphi$ be a solution for TU-games such that $\varphi(N, v) \in$ $\mathscr{C}(N, v)$ for convex games (e.g., the nucleolus or the Shapley value). So, we have that $\varphi\left(N, v_{f_{\alpha}}\right) \in \mathscr{C}\left(N, v_{f_{\alpha}}\right)$ for every $\alpha \in[E / C, 1]$.

It is important that a bankruptcy allocation resulting from O'Neill's bankruptcy game generalization proposed before belongs to the core of the game. If this situation does not occur, there exist at least a pair of agents such that their assigned amount is less than the remaining estate after satisfying the claims of the other agents according to $\alpha$, and we think that this will be an undesirable allocation. That is the reason we are considering the set of bankruptcy games $\mathscr{W}^{B}$ and a solution for TU-games such that it belongs to the core of the game.

By Theorem $6, \varphi\left(N, v_{f_{\alpha}}\right)$ is a solution to the problem $(E ; c)$ for every $\alpha \in[E / C, 1]$. Now, we establish the next expression for every bankruptcy problem $B=(E ; c)$ and every agent $i \epsilon$ $N$ :

$$
\rho_{i}^{\varphi}(B)=\frac{1}{1-E / C} \int_{E / C}^{1} \varphi_{i}\left(v_{f_{\alpha}}\right) d \alpha
$$

Proposition 8. $\rho^{\varphi}(B)=\left(\rho_{1}^{\varphi}(B), \ldots, \rho_{n}^{\varphi}(B)\right)$, with $\rho_{i}^{\varphi}(B)$ as in (34) being a solution to the bankruptcy problem $B=(E ; c)$.

Proof. $\varphi_{i}\left(N, v_{f_{\alpha}}\right)$ is a solution to $(E ; c)$. Then,

$$
0 \leq \varphi_{i}\left(N, v_{f_{\alpha}}\right) \leq c_{i}, \quad \alpha \in\left[\frac{E}{C}, 1\right],
$$

and, because of integral monotonicity,

$$
\rho_{i}^{\varphi}(B) \leq c_{i} .
$$

Even more, $\varphi\left(N, v_{f_{\alpha}}\right) \in \mathscr{C}\left(N, v_{f_{\alpha}}\right)$ implies that the sum of its entries is equal to $E$ and, then,

$$
\sum_{i=1}^{n} \rho_{i}^{\varphi}(B)=\frac{1}{1-E / C} \int_{E / C}^{1} \sum_{i=1}^{n} \varphi_{i}\left(N, v_{f_{\alpha}}\right) d \alpha=E .
$$

The bankruptcy solution given by (34) is an expected value of the solutions $\varphi$ taking a uniform distribution over the set of generalized O'Neill's bankruptcy games with a nonempty core. It is not our intention to provide a characterization or a full study of the properties of solution (34): we are only showing a way to use the results for providing new bankruptcy solutions.

\section{Appendix}

\section{Proof of Lemmas}

Proof of Lemma 1. First, we prove that, for every $\tau_{1}, \tau_{2}, \tau_{3} \in$ $[0, C]$ such that $\tau_{1}+\tau_{2}+\tau_{3} \leq C$, we have

$$
f\left(\tau_{2}+\tau_{3}\right)-f\left(\tau_{1}\right) \leq f\left(\tau_{1}+\tau_{2}+\tau_{3}\right)-f\left(\tau_{1}+\tau_{2}\right) .
$$

With $\tau_{2}=0$ we have an equality. Assume $\tau_{2}>0$ and recall that if $f$ is a convex function on $[a, b]$ and $a<u_{1}<u_{2}<u_{3}<b$, then

$$
\frac{f\left(u_{2}\right)-f\left(u_{1}\right)}{u_{2}-u_{1}} \leq \frac{f\left(u_{3}\right)-f\left(u_{1}\right)}{u_{3}-u_{1}} \leq \frac{f\left(u_{3}\right)-f\left(u_{2}\right)}{u_{3}-u_{2}} .
$$


If $\tau_{3}=\tau_{2}$ then $\tau_{1}<\tau_{1}+\tau_{2}<\tau_{1}+\tau_{2}+\tau_{3}$, and using (A.2) we have

$$
\frac{f\left(\tau_{1}+\tau_{3}\right)-f\left(\tau_{1}\right)}{\tau_{3}} \leq \frac{f\left(\tau_{1}+\tau_{2}+\tau_{3}\right)-f\left(\tau_{1}+\tau_{2}\right)}{\tau_{3}} .
$$

In the same way, if $\tau_{3}>\tau_{2}$ then $\tau_{1}<\tau_{1}+\tau_{2}<\tau_{1}+\tau_{3}<$ $\tau_{1}+\tau_{2}+\tau_{3}$. So,

$$
\begin{aligned}
\frac{f\left(\tau_{1}+\tau_{3}\right)-f\left(\tau_{1}\right)}{\tau_{3}} & \leq \frac{f\left(\tau_{1}+\tau_{3}\right)-f\left(\tau_{1}+\tau_{2}\right)}{\tau_{3}-\tau_{2}} \\
& \leq \frac{f\left(\tau_{1}+\tau_{2}+\tau_{3}\right)-f\left(\tau_{1}+\tau_{2}\right)}{\tau_{3}}
\end{aligned}
$$

and, if $0<\tau_{3}<\tau_{2}$ then $\tau_{1}<\tau_{1}+\tau_{3}<\tau_{1}+\tau_{2}<\tau_{1}+\tau_{2}+\tau_{3}$, and consecutively

$$
\begin{aligned}
\frac{f\left(\tau_{1}+\tau_{3}\right)-f\left(\tau_{1}\right)}{\tau_{3}} & \leq \frac{f\left(\tau_{1}+\tau_{2}\right)-f\left(\tau_{1}+\tau_{3}\right)}{\tau_{2}-\tau_{3}} \\
& \leq \frac{f\left(\tau_{1}+\tau_{2}+\tau_{3}\right)-f\left(\tau_{1}+\tau_{2}\right)}{\tau_{3}} .
\end{aligned}
$$

These facts imply that (A.1) is true, specifically for $\tau_{1}=c(S)$, $\tau_{2}=c(T \backslash S)$, and $\tau_{3}=c_{i}$, where $S \subseteq T \subseteq N \backslash\{i\}$. The lemma follows assigning the previous values to $\tau_{1}, \tau_{2}$ and $\tau_{3}$; rewriting (A.1) we have

$$
v_{f}(S \cup\{i\})-v_{f}(S) \leq v_{f}(T \cup\{i\})-v_{f}(T) .
$$

Proof of Lemma 2. From its definition, $f^{*}$ is continuous and satisfies $f^{*}(0)=0, f^{*}(C)=E$. For $\tau_{1}, \tau_{2} \in[0, C]$ such that $0 \leq \tau_{1} \leq \tau_{2} \leq C$ we have

$$
f^{*}\left(\tau_{1}\right)-f^{*}\left(\tau_{2}\right)=f\left(C-\tau_{2}\right)-f\left(C-\tau_{1}\right) \leq 0 .
$$

So, $f^{*}$ is an increasing function and thus $f^{*} \in \mathscr{F}^{B}$. In addition, for every $\tau \in[0, C]$,

$$
\begin{aligned}
\left(f^{*}\right)^{*}(\tau) & =f(C)-f(C-C)-f(C)+f(C-C+\tau) \\
& =f(\tau)
\end{aligned}
$$

The second statement of the lemma follows from the equation

$$
\begin{aligned}
v_{f}^{*}(S) & =v_{f}(N)-v_{f}(N \backslash S)=f(C)-f\left(C-\sum_{i \in S} c_{i}\right) \\
& =f^{*}\left(\sum_{i \in S} c_{i}\right)=v_{f^{*}}(S) .
\end{aligned}
$$

Now, supposing $f$ is convex, we have, for every $t \in[0,1]$ and $\tau_{1}, \tau_{2} \in[0, C]$,

$$
\begin{aligned}
t f^{*} & \left(\tau_{1}\right)+(1-t) f^{*}\left(\tau_{2}\right) \\
& =t\left(f(C)-f\left(C-\tau_{1}\right)\right)+(1-t)\left(f(C)-f\left(C-\tau_{2}\right)\right) \\
& =f(C)-\left(t f\left(C-\tau_{1}\right)+(1-t) f\left(C-\tau_{2}\right)\right) \\
& \leq f(C)-f\left(t\left(C-\tau_{1}\right)+(1-t)\left(C-\tau_{2}\right)\right) \\
& =f(C)-f\left(C-\left(t \tau_{1}+(1-t) \tau_{2}\right)\right) \\
& =f^{*}\left(t \tau_{1}+(1-t) \tau_{2}\right)
\end{aligned}
$$

It follows that $f^{*}$ is concave. If $f$ is concave, the previous inequality is reversed and we obtain the convexity of $f^{*}$. These facts prove the third statement of the lemma.

Finally, if $f(\tau) \leq g(\tau)$ for every $\tau \in[0, C]$, then

$$
f^{*}(\tau)=f(C)-f(C-\tau) \geq g(C)-g(C-\tau)=g^{*}(\tau),
$$

and, if $f^{*}(\tau) \geq g^{*}(\tau)$ then $f^{*}(C-\tau) \geq g^{*}(C-\tau)$, so $f(C)-f(\tau) \geq g(C)-g(\tau)$. Thus, $g(\tau) \geq f(\tau)$. Changing the corresponding inequality signs, the proof is complete.

\section{Conflict of Interests}

The authors declare that there is no conflict of interests regarding the publication of this paper.

\section{Acknowledgment}

The authors acknowledge support from CONACyT Grant 167924.

\section{References}

[1] B. O'Neill, "A problem of rights arbitration from the Talmud," Mathematical Social Sciences, vol. 2, no. 4, pp. 345-371, 1982.

[2] I. J. Curiel, M. Maschler, and S. H. Tijs, "Bankruptcy games," Zeitschrift für Operations Research, vol. 31, no. 5, pp. 143-159, 1987.

[3] N. Dagan and O. Volij, "The bankruptcy problem: a cooperative bargaining approach," Mathematical Social Sciences, vol. 26, no. 3, pp. 287-297, 1993.

[4] W. Thomson, "Axiomatic and game-theoretic analysis of bankruptcy and taxation problems: a survey," Mathematical social sciences, vol. 45, no. 3, pp. 249-297, 2003.

[5] T. Driessen, "The greedy bankruptcy game: an alternative game theoretic analysis of a bankruptcy problem," in Game Theory IV (Year-Book "Theory of Games and Applications"), L. A. Petrosjan and V. V. Mazalov, Eds., pp. 45-61, Nova Science, New York, NY, USA, 1998.

[6] H. P. Young, "Distributive justice in taxation," Journal of Economic Theory, vol. 44, no. 2, pp. 321-335, 1988.

[7] L. S. Shapley, "A value for n-person games," in Contributions to the Theory of Games, H. W. Kuhn and A. W. Tucker, Eds., vol. 2 of Annals of Mathematical Studies no. 28, pp. 307-317, Princeton University Press, Princeton, NJ, USA, 1953. 
[8] A. S. Nowak and T. Radzik, "A solidarity value for $n$-person transferable utility games," International Journal of Game Theory, vol. 23, no. 1, pp. 43-48, 1994.

[9] L. S. Shapley, "Cores of convex games," International Journal of Game Theory, vol. 1, no. 1, pp. 11-26, 1971. 

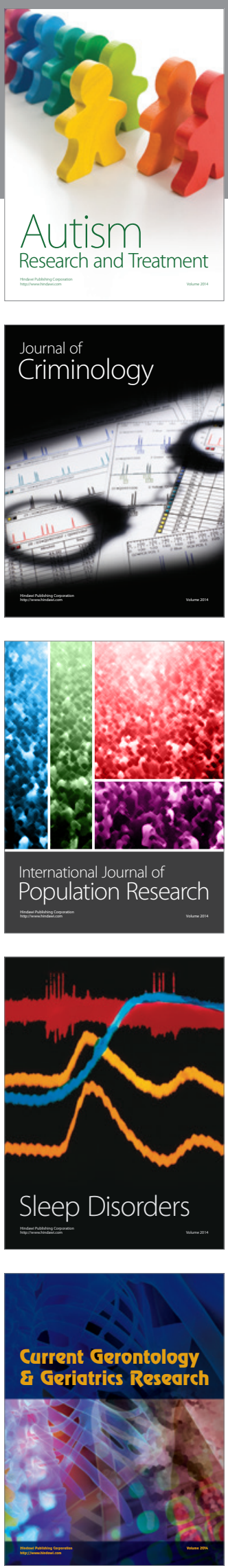
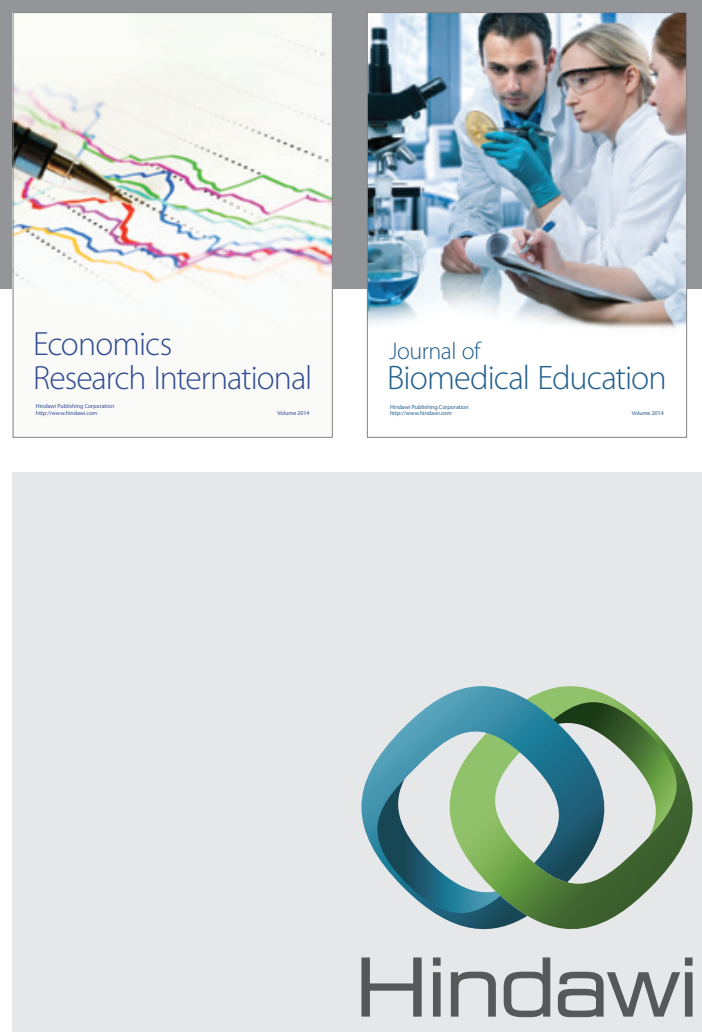

Submit your manuscripts at

http://www.hindawi.com
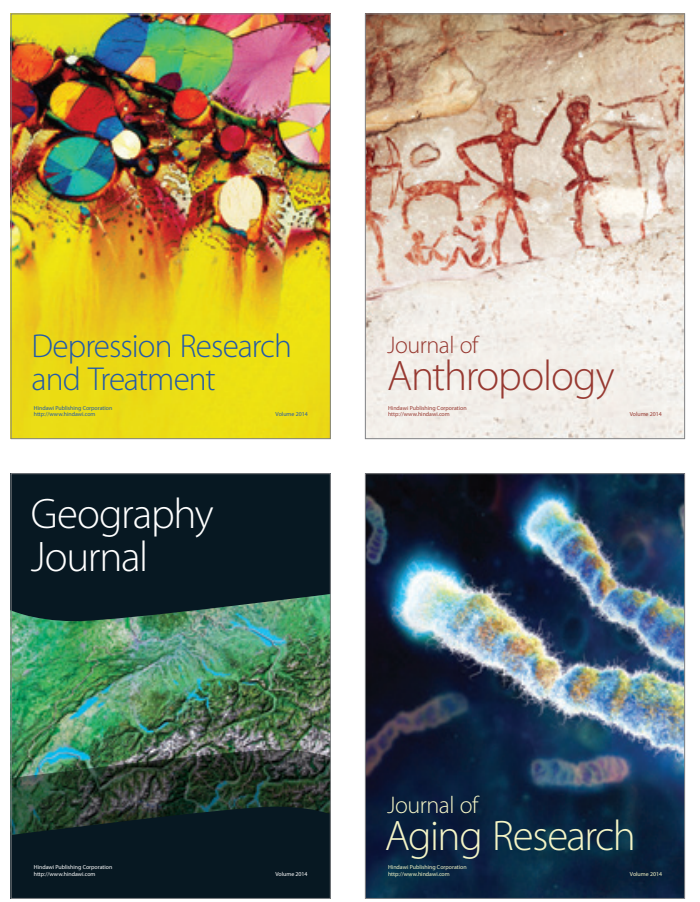
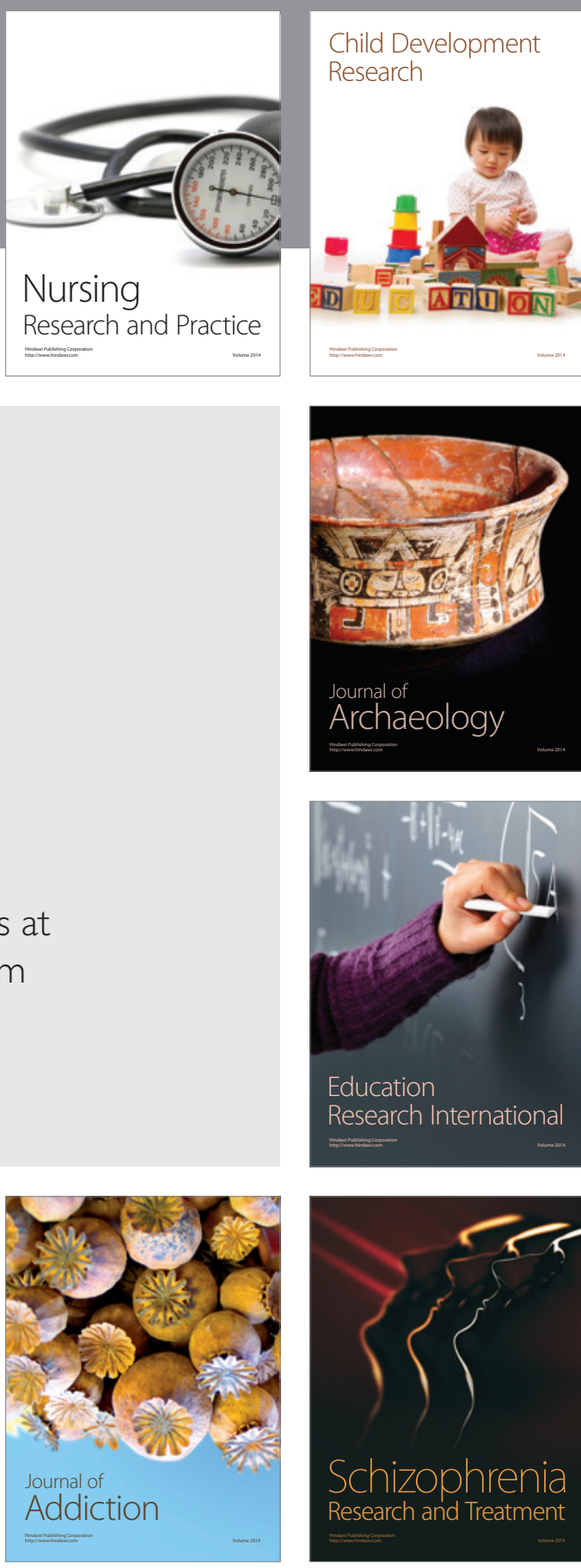

(D)
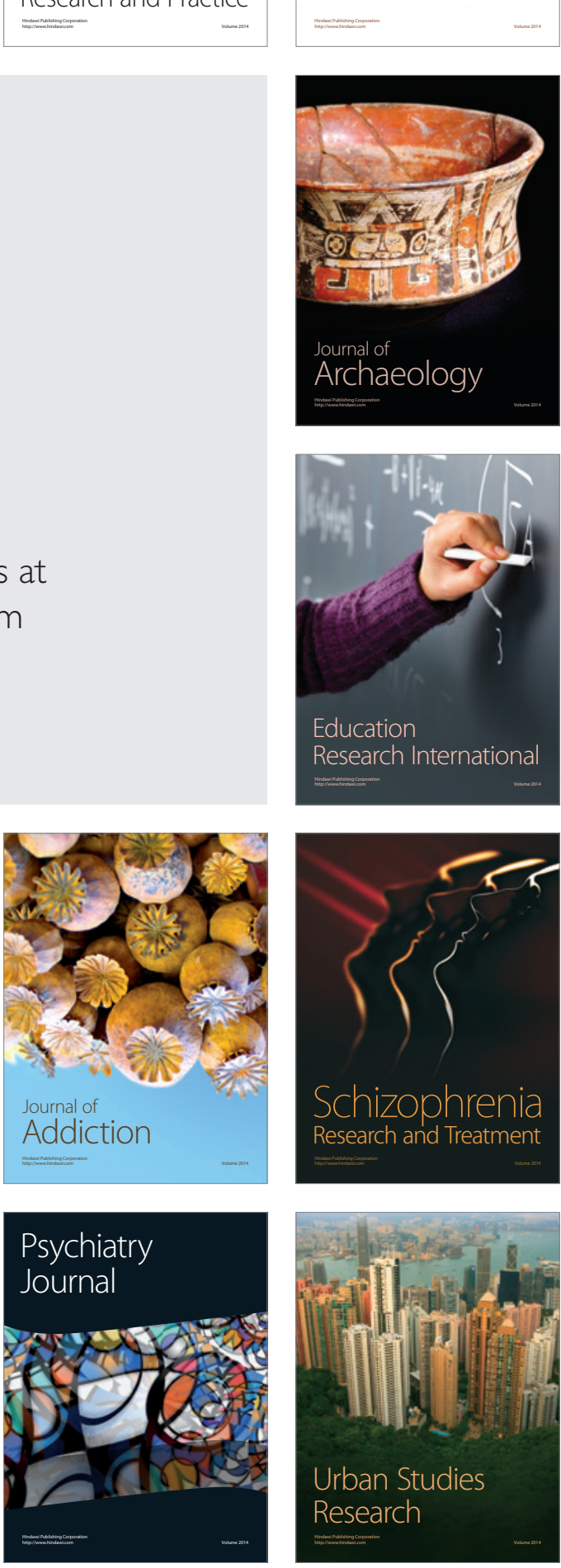